\title{
Graphical Environment to Study Reliability of Electric Power Distribution Systems
}

\author{
José L. Bernal-Agustín ${ }^{1}$, Rodolfo Dufo-López ${ }^{1}$, Héctor Ortiz-de-Landázuri-Díaz ${ }^{1}$, José M. Yusta-Loyo ${ }^{1}$ \\ Javier Contreras ${ }^{2}$, José A. Domínguez-Navarro ${ }^{1}$, C. Monteiro ${ }^{3}$, Franklin Mendoza ${ }^{4}$ \\ ${ }^{1}$ Department of Electrical Engineering - CPS, Zaragoza University. Calle María de Luna, 3, 50018 Zaragoza (Spain) \\ Phone:+34 976 761921, fax:+34 986812601 \\ e-mail: jlbernal@unizar.es, rdufo@unizar.es, ortizdiazhector@yahoo.es,jmyusta@unizar.es, jadona@unizar.es
}

${ }^{2}$ E.T.S. de Ingenieros Industriales, Castilla-La Mancha University. Campus Universitario s/n, 13071 Ciudad Real (Spain) Phone: +34 926 295464, fax: +34 926 295361, e-mail: Javier.Contreras@uclm.es

${ }^{3}$ INESC Porto, Unidade de Energia. Praça da República, n. ${ }^{\circ} 93.4050$ - 497 Porto (Portugal) Phone:.+351 22209 4230, fax. +351 22208 4172, e-mail: cmonteiro@inescporto.pt

${ }^{4}$ Departamento de Ingeniería Eléctrica - Universidad Nacional Politécnica Experimental Antonio José de Sucre. Puerto Ordaz, Estado Bolívar (Venezuela), e-mail: fmen@cantv.net

\begin{abstract}
.
In this paper we present an application for reliability studies of electric power distribution systems. These studies are easily carried out using a graphical interface that allows interaction with the user. The program is capable of evaluating a reliability index and allows the modification of features related to the distribution network or related to the way in which service restoration is done when there is a fault in the system. Thus, it is possible to study several reliability improvement measures as shown in an example in this paper.
\end{abstract}

\section{Key words}

Reliability indexes, Electric Power Distribution System.

\section{Introduction}

The power quality in electric power distribution systems is usually measured using levels which are compared to reference or limit levels $[1,2]$. If the power quality levels are lower than the reference or limit levels, companies may be penalised. Several countries have applied this power quality control system. For example, Argentina has a very complicated power quality control system [3]. The quality levels of every customer of the distribution companies are measured and monitored, so utilities may be penalised if their individual quality level is lower than a minimum. Other countries have implemented similar but less sophisticated power quality regulation schemes. For instance, in Spain minimum global quality levels are imposed to distribution companies [4]. They are measured as average levels in various zones with homogeneous characteristics.

In this paper, a graphical environment (SCIndex) is presented. It is a useful tool for the study of the reliability of power distributions systems. The developed tool allows simulating all the steps, since the beginning of the fault until it is eliminated and the supply of electric power is restored to the consumers [5]. Also, the program calculates an index of reliability (ASIDI) used for the measurement of the power quality of the system. The user can easily modify the distribution network; for example, he can add new switches; add lines, add more brigades, etc., evaluating the possible improvement in the power quality of the system. Therefore, this software is a useful tool for electric power distributions companies.

\section{Reliability indexes}

The system continuity of supply level is controlled through system indices. The system indices used in Spain are:

Average System Interruption Frequency Index (ASIFI or NIEPI, in Spanish).

NIEPI is the installed capacity equivalent number of interruptions at medium voltage $(1 \mathrm{kV} \leq \mathrm{V} \leq 36 \mathrm{kV})$.

Interruption Duration Index (ASIDI or TIEPI, in Spanish).

TIEPI is defined as the installed capacity equivalent interruption time at medium voltage $(1 \mathrm{kV} \leq \mathrm{V} \leq 36 \mathrm{kV})$. These indexes are defined by the following expressions:

$$
N I E P I=\frac{\sum_{i=1}^{N} I C_{i}}{\sum I C}
$$

TIEPI $=\frac{\sum_{i=1}^{N} H_{i} x I C_{i}}{\sum I C}$ 
where:

$\sum$ IC Total sum of the installed capacity in the distributor's MV/LV transformer stations plus the capacity contracted at MV (in kVA).

$\mathrm{IC}_{\mathrm{i}}$ Installed capacity in the distributor's MV/LV transformer stations plus the capacity contracted at $\mathrm{MV}$, affected by interruption «i» lasting $\mathrm{H}_{\mathrm{i}}$ (in kVA).

$\mathrm{H}_{\mathrm{i}} \quad$ Time of the interruption to supply affecting capacity $\mathrm{PI}_{\mathrm{i}}$ (in hours).

$\mathrm{N}$ Total number of interruptions during the period under consideration.

The interruptions to be taken into account when calculating the TIEPI shall be those lasting longer than three minutes.

In the work presented in this paper, only the TIEPI index for measurement of the reliability has been considered. In future works the NIEPI will be calculated too.

\section{Graphical environment}

The graphical environment (SCIndex) has been developed using Visual Basic (the calculation effort is small).

SCIndex makes use of toolbars for easy access to its features. The toolbars contain several symbols, each of which corresponds to elements of power distribution systems.

The user can draw power distribution systems using typical cables used by distribution companies in Spain. Also, the user can modify the power distribution system and simulate the full process of location and elimination of faults.

The software calculates the TIEPI associated to the process mentioned above. Finally, the program generates a report that contains all the steps and results of the simulation. We show the program functionalities in Fig. 1.


Fig. 1. Scheme of the program functionalities.

\section{Example}

An example of a typical urban power distribution system is presented.

The urban distribution networks are usually underground and they have a meshed topology with radial exploitation. The proposed case is shown in Fig. 2. It is a network with two substations that feed five $\mathrm{MV} / \mathrm{LV}$ transformer stations $(\mathrm{CT} 6=630 \mathrm{kVA}, \mathrm{CT} 8=600 \mathrm{kVA}$, $\mathrm{CT} 3=800 \mathrm{kVA}, \mathrm{CT} 4=400 \mathrm{kVA}, \mathrm{CT} 7=600 \mathrm{kVA})$.

The distribution company is studying how to place a node between disconnecting switches 12 and 13 to connect this node to node 4 through a switch, thus creating two possible supply routes for the MV/LV transformer station.
First we obtain the value of the TIEPI index with the network in its initial state. Then, there is a short circuit in the line that connects disconnecting switches 11 and 12 , and when automated switch 6 is fired (the switch in the substation) the power team brigade 3 goes to open disconnecting switch 12. Switch 6 is then closed and fired again, thus the fault is located before the opened disconnecting switch.

With switch 6 open, disconnecting switch 12 is closed and a power team brigade goes to open disconnecting switch 11. Once opened, automated switch 6 is closed and checked that it cannot be fired. Therefore, we have located the fault in the line that connects disconnecting switches 11 and 12. Consequently, the brigade goes to open disconnecting switch 12 (see Fig. 3). Once the simulation is finished, the program provides a TIEPI index value of 1.37 hours. 


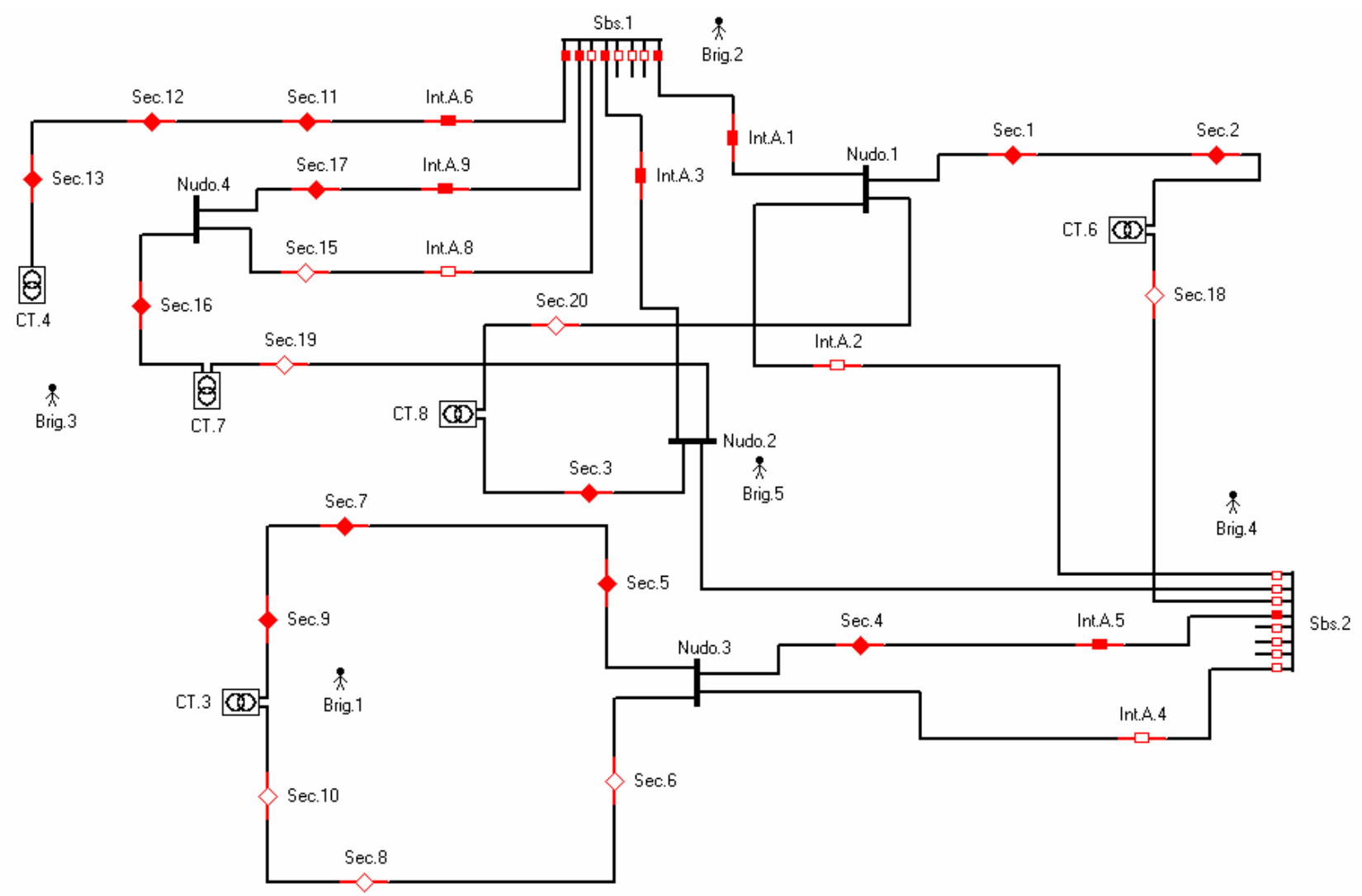

Fig. 2. Urban power distribution system.



Fig. 3. Urban power distribution system with the fault located. 


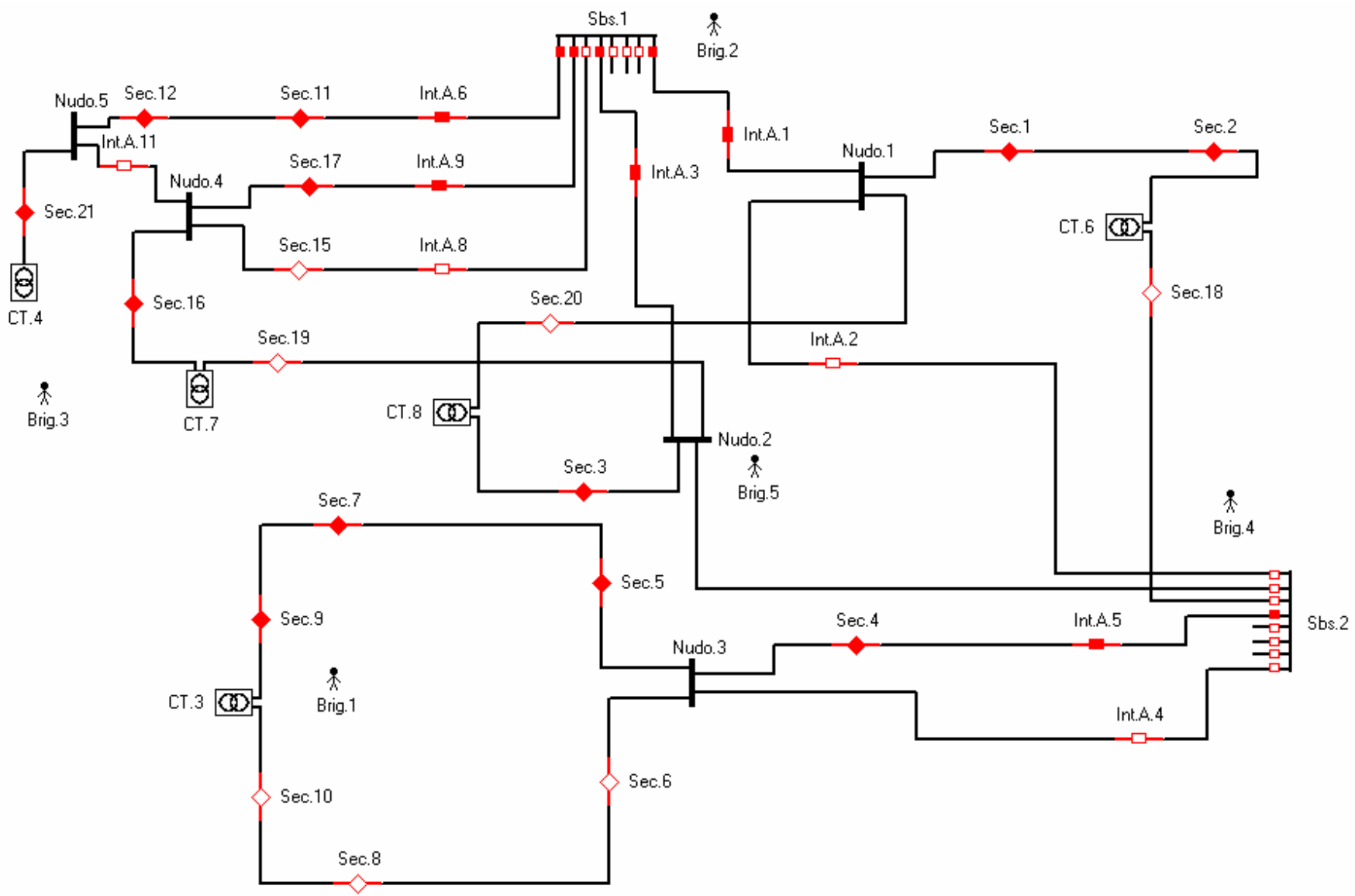

Fig. 4. Network with an additional line between nodes 4 and 5.

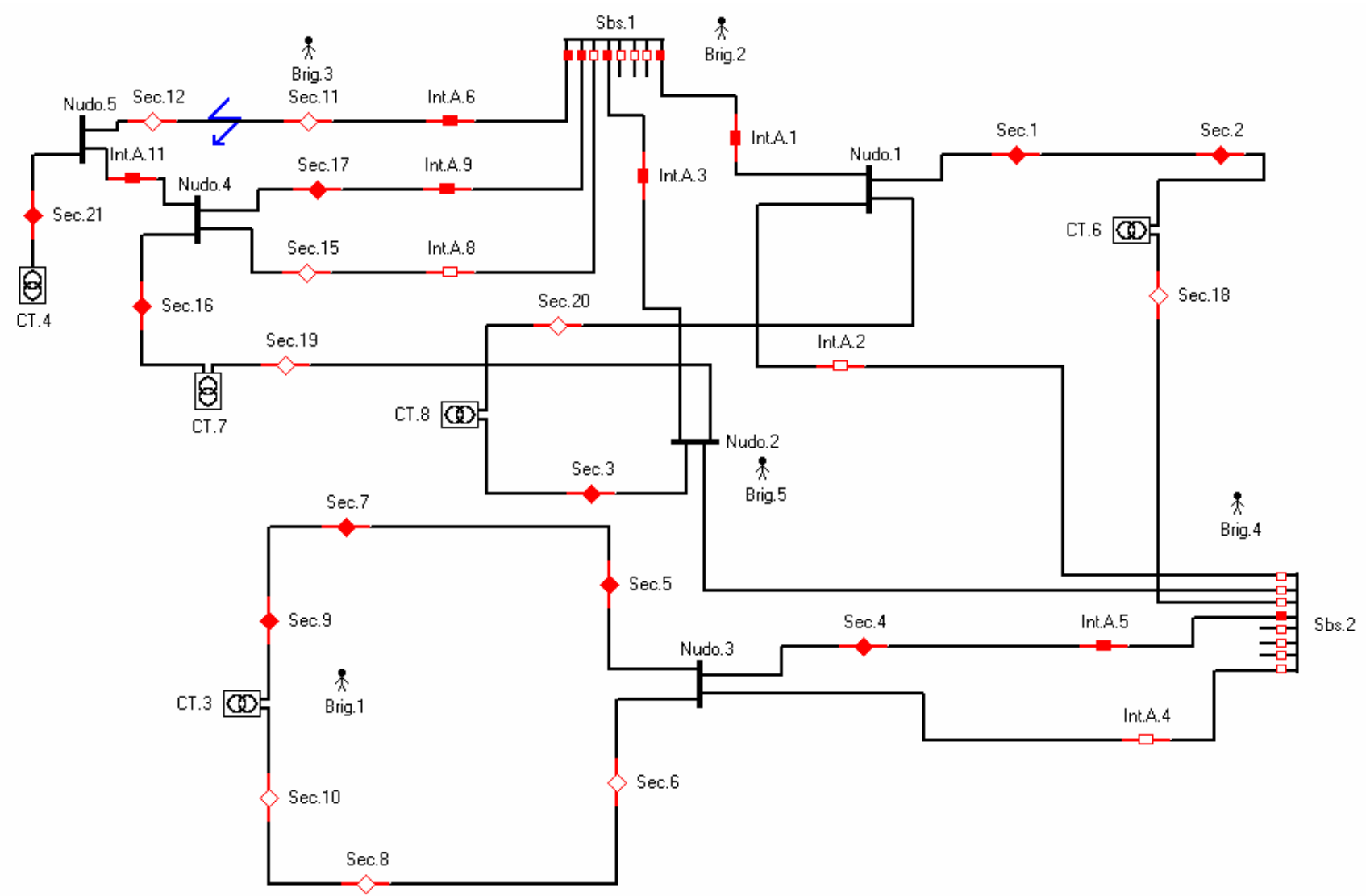

Fig. 5. Final situation with one isolated fault and restoration of supply. 


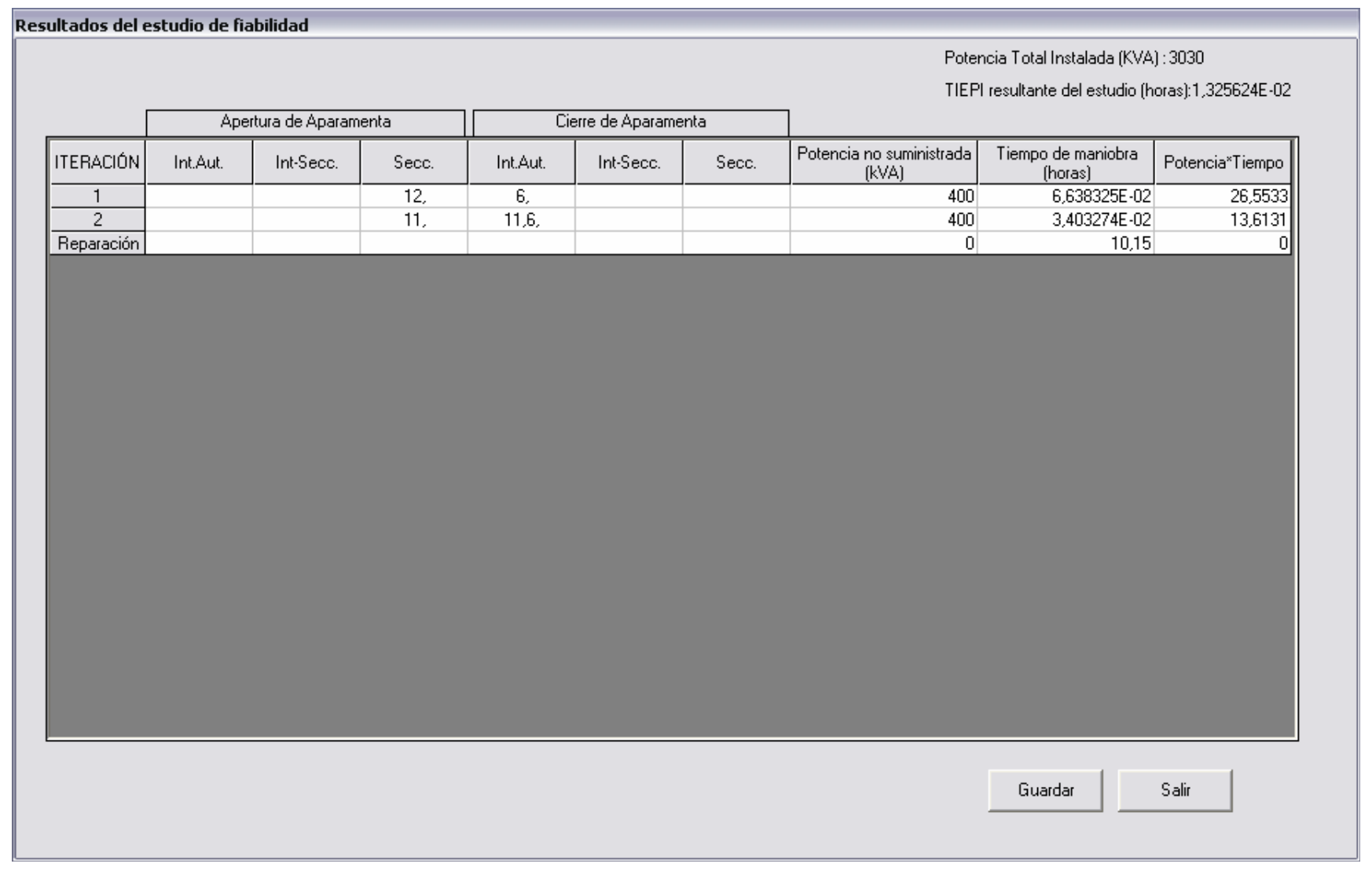

Fig. 6. Table of results.

Now we proceed to manage the fault with the modification previously mentioned (see Fig. 4). The main advantage is that we can create an alternative route to $\mathrm{MV} / \mathrm{LV}$ transformer station 4, guaranteeing the supply in faulty situations.

Again, we simulate a fault in the line connecting disconnecting switches 11 and 12. Thus, the automated switch acts now. Then, the power brigade goes to disconnecting switch 12 and opens it. When closing the head switch, we can observe that it opens again, meaning that the fault is located before the opened disconnecting switch.

Leaving disconnecting switch 12 open, automated switch 12 is closed to feed MV/LV transformer station 4 through node 4. Meanwhile, brigade 3 proceeds to locate and isolate the fault until the final situation is reached as shown in Fig. 5.

Once the study of the fault has finished we obtain the final results as shown in Fig. 6. We can see in the table that the TIEPI index value for the study is 0.013 hours.

\section{Conclusions}

A useful tool for study of power quality of power distributions systems has been presented. This software can be used by power distribution companies to improve the reliability of their power distribution systems.

\section{Future Developments}

In future works, a genetic algorithm will determine the actions to improve power quality. Other reliability indices, such as the NIEPI, will be calculated and used for evaluating the power quality of power distribution systems.

\section{References}

[1] Román, J., Gómez, T., Muñoz, A., Peco, J., "Regulation of distribution network business", IEEE Trans. On Power Delivery, Vol 14, April 1999. pp 662-669.

[2] Rivier, J., de la Fuente, I., Gómez, T., Román, J., "Power Quality regulation under the new regulatory framework of distribution systems", $13^{\text {th }}$ PSCC, Trondheim, Norway, June 1999, Vol. 2, pp. 891897.

[3] Argentinean Ministry of Economy and Industry "Public internacional auction of Edenor S.A. and Edesur S.A. - Sub annexe 4: Power Quality Norms and penalisations" 1992 (in Spanish).

[4] Royal Decree 1955/2000, dated December 1st, regulating transmission, distribution, trading and supply activities and authorisation procedures for electric power installations (in Spanish).

[5] Rivier Abbad, J., "Calidad del servicio. Regulación y optimización de inversiones". Tesis Doctoral. Universidad Pontificia Comillas de Madrid. 1999. 